\title{
Chemical Vapor Deposition Based Superelastic and Superhydrophoboic Thermoplastic Polymeric Nanofibrous Aerogels for Water Purification
}

Jianwei Lu (D 1000005539@ujs.edu.cn )

Yangang Jiang

Ru Xiao

Karl I Jacob

Lei Tao

Songjun Li

Li Guo

\section{Research Article}

Keywords: nanofibrous aerogels, siloxane coating, superhydrophobic, chemical vapor deposition

Posted Date: February 23rd, 2022

DOI: https://doi.org/10.21203/rs.3.rs-1372348/v1

License: (c) (i) This work is licensed under a Creative Commons Attribution 4.0 International License. Read Full License

Version of Record: A version of this preprint was published at Journal of Inorganic and Organometallic Polymers and Materials on April 13th, 2022. See the published version at https://doi.org/10.1007/s10904-022-02330-z. 


\section{Abstract}

Developing high-performance, low-cost and large-scale absorbent materials is crucial for the treatment of water pollution caused by pollutants leakage and emission. Herein, superelastic and superhydrophobic thermoplastic polymeric nanofibrous aerogels (NFAs) were created for removal pollutants from water by using a facile and effective method. Poly(vinyl alcohol-co-ethylene) (EVOH) nanofibers fabricated by mass-production techniques were used to construct three-dimensional NFAs through combing freezedrying process and cross-linking treatment. The optimal parameters for creating EVOH NFAs with good formability and resilience, including composition and ratio of dispersion, dosage of cross-linking agent were obtained through experiments. EVOH nanofibers bonded with each other by glutaraldehyde under acidic conditions to from fibrous network structure in EVOH NFAs. The silane-coated EVOH NFAs were prepared through further modification with vapor-phase methyltrichlorosilane. The deposition of siloxane improved mechanical strength and decreased plastic deformation after 500 cyclic compressions. An asperate fibrous and granular siloxane coating was deposited on the surface of EVOH NFAs. The surface water contact angle increased from $104.4 \pm 4.0^{\circ}$ to $152.7 \pm 1.9^{\circ}$, wettability of NFAs transitioned to being superhydrophobic. Silane-coated EVOH NFAs exhibited superior absorption capacity $(40-92 \mathrm{~g} / \mathrm{g})$ for a variety of organic pollutants. The organic pollutants would be collected and the sorbents could be reused after distillation or squeezing. A successful scale-up of such materials open up a new insight into design polymeric aerogels in low-cost and large-scale with substantial industrial water purification applications.

\section{Introduction}

One of the greatest challenges afflicting people is insufficient access to clean water [1-3]. Aquatic pollution caused by oil and organic solvents from chemicals spills pose a huge threat to human health and global environment $[4,5]$. With increasing human population, there is increasing demand for developing simple, efficient and economical strategies to extract water by decontaminating water from wastewater. Various traditional techniques, that v combustion [6], chemical treatment [7], absorption [8] and bioremediation [9] have been widely investigated for water purification. Usually, the use of combustion and chemical treatments would be accompanied by "side effects" and face the problems of secondary pollution [10], bioremediation has the problems of time-consuming and uncontrollable processes [11], limiting their practical applications. The absorption method is regarded as one of the most effective method for removing pollutants from water $[12,13]$. Various sorbent materials were developed for absorbing oils and organic chemicals [14-16]. However, slow separation, poor efficiency and high prices of sorbent materials still limit their general usage in the separation process.

Nanofibrous aerogel (NFA) is one kind of three-dimensional (3D) materials constructed by assembly of nanofibers, which possess favorable features such as low density, high porosity, large specific surface area, good pore interconnectivity and controllable shape [17-19], which have been attracting significant interests in the filed of oil-water separation. Notably, as far as we know, most of the studies on NFAs were based on cellulose nanofibers as the building blocks. However, the vast majority of nanofiber types are incapable of forming a stable sol-gel system, which could be often associated with the preparation of 
cellulose NFAs. Therefore, several methods for preparing cellulose NFAs are not applicable to other nanofibers $[2,20]$. Moreover, nanofibers currently used in fabrication of NFAs were mostly obtained through high-cost and low-output strategies, which severely hindered aerogels' industrial absorption applications [21, 22]. Therefore, the design and creation of low-cost easily moldable NFAs as highperformance absorbent materials through an easily accessible and gelation-free method is of significant interest.

In recent years, with the continuous exploration of fabrication strategies for aerogels, a versatile and gelation-free process was developed for generating NFAs. Si [23] et al. designed a method to create ultralight and elastic aerogel sorbents by combining the freeze-drying process and the chemical crosslinking technique. The skeleton of resultant NFAs was constructed by the assembly of electro-spun polyacrylonitrile nanofibers and silicon dioxide $\left(\mathrm{SiO}_{2}\right)$ nanofibers, and a bifunctional benzoxazine was used to bond the fibrous networks. Fu [24] et al. dispersed poly(ethylene-co-polyvinyl alcohol) (EVOH) particles, polyphosphoric acid (PPA) and $\mathrm{SiO}_{2}$ nanofibers into water/tert-butanol mixture to form composite nanofibrous suspensions, and obtained composited NFAs through freeze-drying and in situ phosphorylation modification. The $\mathrm{SiO}_{2}$ nanofibers were selected as the building blocks, EVOH was chosen as the binder and PPA was taken as the modifier to construct the phosphorylated 3D composite NFAs. The resultant NFAs could rapidly absorb lysozyme from water. Qian [25] et al. took electro-spun polyimide (PI) nanofibers as skeleton materials for NFAs, and provided PI NFAs superelasticity by thermally induced intermolecular condensation. The PI NFAs exhibited recoverable ultimate strain of $99 \%$. In general, NFAs could be obtained by the dispersion the nanofibers, followed by the freeze-drying and cross-linking treatments. Nevertheless, the difficulties in developing a low-cost, high-efficiency process still remained with the current fabrication process of NFAs. The thermoplastic polymeric nanofibers have been demonstrated to be mass-produced through effective processes, which is based on the phase separation of immiscible thermoplastic polymeric blends with cellulose ester [26]. This mass-production method, called the Melt-Extrusion-Phase-Separation have already been used in the fabrication of a variety of thermoplastic polymeric nanofibers (including polyester [27], polyamide [28] and polyolefin [29]) at lowcost. These thermoplastic polymeric nanofibers could be dispersed into a solution to form suspensions, and NFAs with good integrity were formed by subsequent freeze-drying [30]. The cross-linking modification effectively made NFAs reusable.

The NFAs can possess a variety of functional properties through various modification treatments, which is highly beneficial to expand their applications [31, 32]. Lu [32] et al. immersed magnetic carbonaceous aerogel into an ethanol solution of $1 \mathrm{H}, 1 \mathrm{H}, 2 \mathrm{H}, 2 \mathrm{H}$-Perfluorooctyltriethoxysilane, then washed and dried to fabricate superhydrophobic aerogels. The resulting NFAs showed robust superhydrophobicity, superoleophilicity, and excellent absorption capacity $(61-113 \mathrm{~g} / \mathrm{g})$ for various liquids. Sai [33] et al. modified bacterial cellulose NFAs through reacting with trimethylchlorosilane in liquid phase to form a hydrophobic coating, which could find applications in the field of oil/water separation. The NFAs can also be modified with vapor phase treatments, with the advantages that no liquid chemistry is needed and no liquid waste is produced [34]. Korhonen [35] et al. deposited a nano-scale titanium dioxide $\left(\mathrm{TiO}_{2}\right)$ coating 
on the surface of cellulose NFAs by using atomic layer deposition to form reusable and recyclable oil absorbents. Besides, the $\mathrm{TiO}_{2}$-coated NFAs also exhibited good photocatalytic activities, being able to decompose methylene blue.

Herein, we reported a versatile, gelation-free and readily accessible method to create absorbent materials based on thermoplastic polymeric nanofibers-EVOH nanofibers by combining freeze-shaping and chemical modifications. The optimum parameters for preparing EVOH NFAs were obtained through experimental screening, and the bonding mechanism between EVOH nanofibers was analyzed. The chemical vapor deposition resulted in a fibrous and granular siloxane coating on the surface of NFAs, and could alter the surface wettability to superhydrophobicity. The silane-coated EVOH NFAs exhibited good oil/water selectivity, remarkable absorption capacity for various liquids and outstanding reusability. The NFAs presented in this work offer a straightforward strategy to construct absorbent materials with good potential for pollutant removal from water and environmental protection.

\section{Experimental Part}

\subsection{Materials}

Poly(ethylene-co-polyvinyl alcohol) (EVOH) resin (38 mol\% ethylene) with a density of $1.17 \mathrm{~g} / \mathrm{cm}^{3}$ and cellulose acetate butyrate ester (CAB, Model: 381 - 20) were obtained from Nippon Gohsei (Japan) and Eastman Chemical Company (United States), respectively. Acetone, acetic acid (99.5\%), glutaraldehyde aqueous solution (GA, $25 \mathrm{wt} \%$ ) and tert-butanol (t-BuOH, $>98 \%$ ) were purchased from Sinopharm Chemical Reagent Co., Ltd. (China). Methyltrichlorosilane (MTS) and most organic solvents were acquired from Sigma-Aldrich Co., Ltd (United States), including phenoxin, hexane, and tetrahydrofuran. Deionized water was used for all experiments. All chemical reagents were used as received.

\subsection{Preparation of EVOH NFAs}

To synthesize EVOH nanofibrous aerogels (NFAs), EVOH nanofibers prepared through Melt-ExtrusionPhase-Separation $[26,36]$ were used as building blocks. Typically, $1 \mathrm{~g}$ EVOH nanofiber bundles were uniformly dispersed in $100 \mathrm{~mL}$ dispersion (water and t-BuOH mixture with different weight ratio) through homogenizing at $12000 \mathrm{rpm}$ for $30 \mathrm{~min}$ to form a stable EVOH nanofibrous suspension. The cross-linking agent GA was added to obtained nanofibrous suspension under constant stirring to form resultant suspensions. The GA contents were controlled to be around $0,0.25,0.50,0.75,1.00,1.25,1.50$ vol\% relative to the dispersion. Then the $\mathrm{pH}$ value of composite suspensions was adjusted to around 4 through adding acetic acid solution. The nanofibrous suspensions with different GA contents underwent a freezedrying process to gain un-cross-linked EVOH NFAs. These prepared samples were heated at $75^{\circ} \mathrm{C}$ for $4 \mathrm{~h}$ to obtain EVOH NFAs.

\subsection{Fabrication of silane-coated EVOH NFAs}


The silane-coated EVOH NFAs were obtained through chemical vapor deposition (CVD) in a furnace. The EVOH NFA samples and a beaker of $5 \mathrm{~mL}$ MTS were placed in a sealed glass desiccator and heated to $50^{\circ} \mathrm{C}$ for $12 \mathrm{~h}$ to make EVOH NFAs reacting with MTS. Finally, the silane-coated samples were put in a vacuum desiccator remove the remaining MTS and by-product $(\mathrm{HCl})$.

\subsection{Characterization}

The apparent densities of NFAs were calculated by dividing mass by volume. The microstructure and elemental distribution of NFAs were observed by employing a scanning electron microscope (SEM, Hitachi U8230) and energy-dispersive X-ray spectroscopy (EDS, Bruker Quantax 400). The surface chemical composition of NFAs was characterized by using a X-ray photoelectron spectroscopy (XPS, ThermoFisher K-alpha), attenuated total reflection-Fourier transform infrared (ATR-FTIR, Bruker EQUINOX55) and X-ray diffractometer (XRD, RigakuD max-1200). The surface wettability of NFAs was assessed using a contact angle meter (Kruss DSA100) with a water or oil volume of $3 \mu \mathrm{L}$ at room temperature. The compression performances of NFAs were characterized using an Instron (model 5969) fitted with a $100 \mathrm{~N}$ load. Cylindrical NFAs with diameter and height of about 30 and $20 \mathrm{~mm}$ was used. Compressive $\sigma-\varepsilon$ curves with strain of 40,60 , and $80 \%$ were recorded at a $30 \mathrm{~mm} / \mathrm{min}$ strain rate. The cyclic compression performances were measured at a $300 \mathrm{~mm} / \mathrm{min}$ strain rate. The Young's modulus of NFAs was calculated from the linear stage $(\varepsilon<20 \%)$ of $\sigma-\varepsilon$ curves. The heat resistance of NFAs was characterized using a thermogravimetric analyzer (TGA, TA instruments Q50). About 5 mg of NFAs was placed into a quartz crucible and heated from 25 to $700^{\circ} \mathrm{C}$ at a $10^{\circ} \mathrm{C} / \mathrm{min}$ heating rate under nitrogen flow $(20 \mathrm{ml} / \mathrm{min})$. The insulation of NFAs was measured using transient hot-wire instrument (Xiatech Electronics TC3000). Two samples clamped the hot wire and tested at voltage of $0.8 \mathrm{~V}$.

\subsection{Density of NFAs}

The density of NFAs was calculated by using the following equation:

$$
\rho_{0}=W_{0} / V_{0}
$$

Where $\rho_{0}$ is the density of NFAs, $W_{0}$ is the mass of NFAs, $V_{0}$ is the volume of NFAs.

\subsection{Porosity of NFAs}

The porosity of NFAs was calculated by using the following equation:

$$
\eta(\%)=1-\left(\frac{W_{1}}{\rho_{1} \times V_{0}}+\frac{W_{2}}{\rho_{2} \times V_{0}}\right) \times 100 \%
$$


where $\eta$ is the porosity of NFAs, $W_{1}$ and $W_{2}$ are the mass of EVOH nanofibers and siloxane deposition, respectively, $\rho_{1}$ and $\rho_{2}$ are the density of $\mathrm{EVOH}$ nanofibers and siloxane deposition, respectively.

\subsection{Measurements of absorption capacity}

The NFAs ( $20 \mathrm{mg}$ ) were immersed into pure oil/organic solvents. After the NFAs reached saturation, they were then taken out and weighted. The absorption weight capacity of aerogels was calculated by using the following equation:

$$
Q(m / m, \%)=\frac{W-W_{0}}{W_{0}} \times 100 \%
$$

3

where $\mathrm{Q}$ is the absorption capacity of NFAs, is the weight of NFAs after liquid absorption.

When the adsorption volume is taken into account, the absorption volume capacity was calculated by using the following equation:

$$
V / V_{0}(v / V, \%)=\frac{Q \times \rho_{0}}{\rho}
$$

4

where $\mathrm{V}$ is the volume of the absorbate, $\rho$ is the density of absorbrate, respectively.

\section{Results And Discussion}

\subsection{Formation of silane-coated EVOH NFAs}

The silane-coated poly(ethylene-co-polyvinyl alcohol) (EVOH) nanofibrous aerogels (NFAs) were designed based on following three criteria: (1) EVOH nanofibers must uniformly assemble into three-dimensional NFAs, (2) the inherent porous structure of NFAs must not be destroyed during chemical modification processing, (3) the resultant NFAs should exhibit reasonable mechanical properties. To satisfy the first requirement, the mixture of water and tert-butanol $(\mathrm{t}-\mathrm{BuOH})$ with different weight ratio was selected as dispersion medium to form stable suspensions and frozen samples. Low temperature vapor deposition was taken to satisfy the second requirement. The formation of cross-linking bonds between EVOH nanofibers endow EVOH NFAs with elasticity [5], and deposition of flexible Si-O-Si bonds improve NFAs' resilience [30], which can satisfy the third criterion.

The preparation pathway of silane-coated EVOH NFAs is shown in Fig. 1a. EVOH nanofibers fabricated through Melt-Extrusion-Phase-Separation were used to form continuous fibrous network. EVOH 
nanofibers were first homogenized in water/t-BuOH mixture to obtain suspensions. The contact angles of $\mathrm{EVOH}$ nanofibrous membranes and water/t-BuOH mixtures with various $\mathrm{t}-\mathrm{BuOH}$ contents were shown in Fig. S1. The water contact angle (WCA) of EVOH nanofibrous membranes is $108.3 \pm 2.1^{\circ}$, indicating EVOH nanofibers could not be wetted well by pure water. A frequently used dispersing agent-t-BuOH was introduced into water. The contact angle of EVOH nanofibrous membranes decreased steeply, with increasing t-BuOH contents. When the contents of $\mathrm{t}-\mathrm{BuOH} \geq 20 \mathrm{wt} \%$, contact angles of membranes were $0^{\circ}$, representing good wetting of $\mathrm{EVOH}$ nanofibers. $\mathrm{EVOH}$ nanofibrous membranes were dispersed into water/t-BuOH mixture. The nanofibrous suspensions with various $\mathrm{t}-\mathrm{BuOH}$ contents were demonstrated in Fig. S2. It is clearly to observe that the stability of nanofibrous suspensions increased, with increasing t$\mathrm{BuOH}$ contents. The dispersion with $\mathrm{t}-\mathrm{BuOH}$ contents $\geq 15 \mathrm{wt} \%$ resulted in nanofibrous suspensions with good stability, after standing for $10 \mathrm{~min}$. The as-prepared nanofibrous suspensions were subsequently frozen in cryogenic refrigerator. As presented in Fig. S3, the introduction of $\mathrm{t}-\mathrm{BuOH}$ effectively decreased volume expansion of frozen suspensions, but exceeded t-BuOH would result in undesired volume shrinkage. When $\mathrm{t}-\mathrm{BuOH}$ contents is $20 \mathrm{wt} \%$, the frozen suspensions achieved good formability almost without any volume change. Therefore, the optimum content of t-BuOH was $20 \mathrm{wt} \%$, and the stable EVOH nanofibrous suspensions would obtain after $30 \mathrm{~min}$ (Fig. 1b).

Bifunctional glutaraldehyde (GA) was introduced into EVOH nanofibrous suspensions as a cross-linker, and acetic acid was blended to provide acidic condition for subsequent cross-linking. After freeze-drying process, un-cross-linked EVOH NFAs would be heated to form cross-linking network. The EVOH NFAs with different GA contents was shown in Fig. S4. With increasing GA contents, the volume of NFAs decreased. Moreover, exceeded GA would give rise to deformation of EVOH NFAs. This was because EVOH nanofibers are flexible, and tend to generate cross-linking shrinkage. The bonding was obviously observed among EVOH nanofibers (Fig. 1c), endowing EVOH NFAs with elastic resilience. The proposed mechanism of cross-linking EVOH nanofibers and glutaraldehyde is presented in Fig. S5a [37], and the successful cross-linking was confirmed by FTIR spectra (Fig. S5b). As demonstrated in Table S1, the volume of EVOH NFAs decreased from $17.72-18.53$ to $12.36-15.60 \mathrm{~cm}^{3}$, with increasing GA contents from 0 to $0.50 \mathrm{vol} \%$. The mass of NFA samples were almost constant, which would result in higher density. However, with continued GA contents increasing, EVOH NFAs turned into irregular in shape, and the volume and density of EVOH NFAs could not be acquired through calculating. It is obviously that adding $0,0.25,0.50$ vol\% GA to EVOH NFAs could achieve good formability. The compressive performances of EVOH NFAs were estimated through applying an external pressure $(200 \mathrm{~g})$. As shown in Fig. S6, the EVOH NFAs with 0 and 0.25 vol\% GA contents could not recover to their original shape after releasing loading. In turn, the EVOH NFAs with 0.5 vol\% GA contents almost exhibited complete shape recovery. Adding GA to EVOH NFAs could introduce chemical covalent bonds, and improve their resilience [5]. Combined with aerogels' shape and compression performance, the optimum content of GA was 0.50 vol\%.

The EVOH NFAs with 0.50 vol\% GA contents reacted with vapor-phase MTS to obtain silane-coated EVOH NFAs. The silanization of EVOH NFAs occurred at $50^{\circ} \mathrm{C}$, MTS precursors deposited on the surface of 
exposed EVOH nanofibers, and the fibrous and granular siloxane could be clearly observed in Fig. 1d. As shown in Fig. S7, the geometric volume of these NFAs remained nearly constant, NFAs just changed color from white to brown after modification.

\subsection{Morphology and structure of silane-coated EVOH NFAs}

As shown in Fig. 2a and b, at low magnification, NFAs exhibited porous network of fibrous structure before and after chemical modification, indicating that chemical vapor deposition (CVD) has almost no influence on the pristine network structure. This was because the chemical modification is accomplished in the vapor phase at a relatively low temperature which would not destroy the aerogel. Zooming in on the EVOH nanofibers revealed the nanofibers in EVOH NFAs had a relatively smooth surface (Fig. 2c). In contrast, after CVD, the surface of EVOH nanofibers became asperate and the siloxane coatings became visible (Fig. 2d). According to previous investigations, different coatings could be formed, which depended on the silane reaction types (horizontal polymerization, covalent attachment and vertical polymerization) [38-40]. The proposed reactions between EVOH nanofibers and MTS were summarized in Fig. S8, all these types could form hydrophobic coatings on the surface of EVOH nanofibers.

As seen in Fig. 2e, the Si element was relatively uniformly dispersed in NFAs consisting of $\mathrm{C}$ and $\mathrm{O}$ elements. Figure $2 f$ displayed the XPS survey scans for silane-coated EVOH NFAs, Si $2 p$ peaks were detected. The Si $2 p$ spectrum can be deconvoluted into two peaks, which located at 102.68 and 103.07 eV were assigned to Si-C and Si-O, respectively (Fig. S9). However, no Si signal was detected in EVOH NFAs' XPS survey scan (Fig. S10). Two new characteristic absorption peaks at 782 and $1273 \mathrm{~cm}^{-1}$ would be found in ATR-FTIR spectra of silane-coated EVOH NFAs (Fig. 2g), corresponding to the asymmetric stretching vibrations of Si-O-Si and $\mathrm{C}-\mathrm{Si}$, respectively [36]. Taken together, all the results indicated the successful deposition of siloxane on the surface of NFAs. Almost no changes were observed in the XRD pattern of EVOH NFAs before and after CVD (Fig. 2h). The crystallinities of EVOH NFAs before and after silanization were 31.48 and $29.54 \%$, respectively, indicating that CVD treatment would not alter crystallization of NFAs. This was because that the deposition of siloxane would not destroy NFAs' crystalline region.

\subsection{Mechanical properties of NFAs}

NFAs can exhibit good compressibility and resilience through cross-linking, which are very important for their practical applications [41-43], However, NFAs assembled by flexible nanofibers in general showed obvious hysteresis loops and large plastic deformations after large strain cyclic compressions [39]. However, EVOH NFAs could withstand high compression strain and show a resilience of about $100 \%$, although after 500 compression cycles under strain of $60 \%$, an obvious plastic deformation $(24.43 \%)$ was observed (Fig. S11b). This was due to the fact that some of the chemical covalent forces were broken during cyclic operations, leading to decreases in strength [44]. Figure 3a shows the compressive $\sigma-\varepsilon$ curves of silane-coated EVOH NFAs at different strain of 40,60 and $80 \%$. These curves are indicative of typical viscoelastic, energy-dissipative behavior commonly observed in soft and highly deformable materials [45]. Similar to EVOH NFAs, silane-coated EVOH NFAs could sustain large deformation, and 
completely recover to their original shape after unloading (Fig. 3a inset images). The strain is 40,60 and $80 \%$, corresponding to the stress of $7.05,14.48$ and $39.71 \mathrm{kPa}$, respectively. These values were higher than those of EVOH NFAs (4.48囚9.89®23.83 kPa) (Fig. S11a). This was ascribed to the deposition of siloxane could improve the strength of NFAs' pores.

Figure 3b presented the cyclic compressive $\sigma$ - $\varepsilon$ curves of silane-coated EVOH NFAs under strain of $60 \%$. After 500 compression cycles, a plastic deformation (9.88\%) was observed, which is much lower than that of EVOH NFAs (24.43\%), clarifying silane-coated EVOH NFAs displayed better flexibility in their structure. This was attributed to the deposition of highly flexible Si-O-Si bonds on the surface of EVOH NFAs, which could substantially improve the resilience of aerogels [30]. The maximum stress and Young's modulus of silane-coated EVOH NFAs decreased from 14.48 and $10.20 \mathrm{kPa}$ to 12.41 and $6.69 \mathrm{kPa}$, respectively, after 500 cycles (Fig. 3c), indicating good durability and stability of silane-coated samples. Figure $3 \mathrm{~d}$ showed energy dissipation and energy loss coefficient of silane-coated EVOH NFAs as a function of compressive test cycles. In the first compression cycle, the energy dissipation was 2.19 $\mathrm{mJ} / \mathrm{cm}^{3}$ and energy loss coefficient $(\Delta U / U$ ) was 0.72 . Large energy dissipation was related to the viscoelasticity of the nanofibrous structure [23]. Besides a decline in first few compression cycles, no significant decreases in energy dissipation, indicating excellent compression fatigue resistance of silanecoated EVOH NFAs [24]. The resilience and durability silane-coated EVOH NFAs are comparable or superior to that of pure/hybrid polymeric aerogels $[8,46]$.

\subsection{Surface wettability of NFAs}

The surface wettability of NFAs is characterized by contact angle. As shown in Fig. 4a, the original WCA of EVOH NFAs was $140.4 \pm 4.0^{\circ}$, but it still not measured up to the standard of superhydrophobicity $(\theta>$ $150^{\circ}$ ). Moreover, the WCA of EVOH NFAs decreased from $140.0 \pm 4.0^{\circ}$ to $0^{\circ}$ in $27 \mathrm{~s}$, clarifying poor waterproof performance of them. According to the Cassie Model [47], formation of asperate micro- and nano-scale structure could achieve superhydrophobic behavior. The deposition of siloxane coatings created nano-sized asperate structures on the surface of EVOH NFAs in fibrous and granular through CVD (Fig. 1d). The WCA of silane-coated NFAs increased from $140.0 \pm 4.0^{\circ}$ to $152.7 \pm 1.9^{\circ}$, and the initial WCA could remain almost constant at least $900 \mathrm{~s}$ (Fig. 4a), demonstrating that NFAs transitioned to being superhydrophobic. The rough hydrophobic siloxane coatings could reduce the water-solid contact area, prevented more water from contacting NFAs. Moreover, the siloxane deposition with low surface energy on $\mathrm{EVOH}$ nanofibers surface also improved the hydrophobicity of NFAs.

The whole NFAs was immersed into water to measure the hydroscopicity of them. As shown in Fig. 4b, the calculated water absorption capacity and saturation absorption time of EVOH NFAs was $40.61 \mathrm{~g} / \mathrm{g}$ and $8 \mathrm{~min}$, respectively. After siloxane coating, the water absorption capacity dropped down to $33.33 \mathrm{~g} / \mathrm{g}$, while the saturation absorption time increased to $400 \mathrm{~min}$, indicating the improvement in waterproof performance. The chemical stability of silane-coated EVOH NFAs was characterized through immersing them into corrosive medium liquids, salt solution and organic solvent. As shown in Fig. 4c, the WCA of silane-coated EVOH NFAs higher than $148^{\circ}$, demonstrating their excellent corrosion resistance. The stained water droplets could bead up both on the surface and in the internal of silane-coated EVOH NFAs 
(Fig. 4d and f), indicating that CVD could endow exterior and interior of the NFAs with hydrophobicity. This was caused by vapor-phase MTS that could penetrate inside fibrous structure and deposit on the surface of nanofibers. The siloxane coatings could be observed both inside and outside the NFAs from the Fig. S12. Silane-coated EVOH NFAs absorbed oil droplets quickly (Fig. 4f), clarifying good oil/water selectivity of silane-coated NFAs. Therefore, the superhydrophobicity and oleophilicity of silane-coated NFAs ensures their superior oil and organic solvent absorbance from water.

\subsection{Absorption efficiency of NFAs}

Silane-coated EVOH NFAs possessed good sorption selectivity, which could quickly absorb oil and organic solvents from water. As shown in Fig. 5a, a small piece of silane-coated EVOH NFAs was placed in diesel oil and water mixtures, in which the stained diesel oil floated on the surface of water. Silanecoated samples completely absorbed stained diesel oil in $19 \mathrm{~s}$, and the water in the beaker became clean. After removing oil-filled aerogels, no oil releasing was observed, indicating fibrous structure could hold the absorbed oil [48]. Moreover, silane-coated EVOH NFAs also absorbed organic solvents immediately from bottom of water (Fig. 5b), and absorbed aerogels could be left floating on water to separate organic solvents from water.

Silane-coated EVOH NFAs with low density and high porosity could be an efficient absorbent for removing pollutants from water. In order to investigate the absorption performance of silane-coated EVOH NFAs, various liquids commonly used in both industrial and domestic area were used as absorbates. Figure $5 \mathrm{c}$ displayed the absorption capacity was assessed by weight gain. The silane-coated EVOH NFAs showed high absorption efficiency for various liquids, which in range from 4008 to $9185 \%$. The high absorption efficiency might be related to NFAs' low density and high porosity [49]. Table S2 summarized the absorption capacities of a variety of other sorbents. The absorption capacity of silane-coated NFAs were much higher than that of some reported sorbents with higher density and lower density, such as cellulose nanofibril aerogels (2300-4600\%) [50], Ni/carbon aerogels (1318-4877\%) [49], polymers (600-2300\%) [51]. Their absorption efficiency was still lower than that of sorbents, which absorption efficiency up to $34800 \%$ [52]. However, the aerogels preparation method is relatively easy, and the building blocks-EVOH nanofibers is relatively cheap, which make the silane-coated EVOH NFAs fabricated in this work is one of most promising adsorbents. It is clearly observed that silane-coated EVOH NFAs had high absorption capacity for the liquids with high density, indicating that the absorption efficiency for various oils and organic solvents were related to the densities of these liquids. In order to eliminate the effect of liquids' density, the volume absorption efficiency was calculated in Fig. $5 \mathrm{~d}$. The volume absorption efficiency for various liquids ranged from 73.14 to $87.83 \%$, which means at least $12 \%$ of total space was inaccessible to these liquids. This might be because some air was trapped in aerogels [48]. The air bubbles were seen during absorption process, and some of them still stayed in the pores of aerogels. Therefore, silanecoated EVOH NFAs could not achieve 100\% volume absorption efficiency, except volume expansion.

The excellent mechanical properties could provide silane-coated EVOH NFAs with stable recyclability. Distillation and squeezing were used to investigate cyclic absorption properties of aerogels. Distillation is a great selection for gathering liquids with low boiling points, and squeezing is preferred for collecting 
high boiling point, valuable pollutants. Hexane with low boiling point $\left(69^{\circ} \mathrm{C}\right)$ was selected as a model for distillation experiments. Hexane filled NFAs were heated to the boiling point, then the vapor of hexane was released and collected. As shown in Fig. 5e, the net absorption capacity almost did not change over the 10 repetitions, demonstrating stable recyclability of silane-coated EVOH NFAs. Chloroform was chosen as a model for squeezing experiments. After the first cycle, the residual in NFAs increased from 0 to approximate 14 times the original weight (Fig. 5f), which might be caused by incomplete compression of NFAs [53]. The absorption efficiency maintained stable during 10 repetitions. Both methods could transform the pollutants into available resources.

\section{Conclusion}

We have demonstrated that a universal and gelation-free method for design and construction of poly(ethylene-co-polyvinyl alcohol) (EVOH) nanofibrous aerogels (NFAs) with good formability and resilience through combining freeze-drying and cross-linking treatment. In the fabrication process, the dispersion consisted of water and tert-butanol with a weight ratio of $4: 1$; glutaraldehyde aqueous solution was selected as cross-linking agent and the content was $0.5 \mathrm{vol} \%$ relative to the dispersion; acetic acid solution was added to provide acidic conditions for cross-linking. The silane-coated EVOH NFAs were obtained by further modification with vapor-phase methyltrichlorosilane. After siloxane coating, the density of NFAs increased from $12.31-15.53$ to $13.96-15.88 \mathrm{mg} / \mathrm{cm}^{3}$, and almost no changes in porosity. In comparison with EVOH NFAs, silane-coated EVOH NFAs exhibited better mechanical strength, cyclic compressibility, heat resistance and thermal insulation. The fibrous and granular siloxane coatings were deposited on the surface of NFAs, and water contact angle increased from $104.4 \pm 4.0^{\circ}$ to $152.7 \pm$ $1.9^{\circ}$. The silane-coated EVOH NFAs with good oil/water selectivity could be used for removing organic pollutants from water, the mass-based and volume-based absorption capacity ranged from 4008 and $73.14 \%$ to 9185 and $87.83 \%$, respectively. The sorbents showed outstanding reusability and reproducibility. We expected that the NFAs with simple modification created through versatile and gelation-free method will open up designs for low-cost, large-scale production of subsequent absorbent materials with water purification.

\section{Author Information}

\section{Declarations}

\section{Acknowledgements}

This work was supported by National Natural Science Foundation of China (No. 51803081 and No. 20874010) and Changzhou Sci\&Tech Program(No .CJ20210042)

\section{References}


1. Y.Q. Wang, Y. He, Y. Fan, H.J. Li, H. Yu, J. Yu, Y.L. Nie, S.H. Wang, A robust anti-fouling multifunctional aerogel inspired by seaweed for efficient water purification. Sep. Purif. Technol. 259, 118153 (2021)

2. F. Wang, J.W. Dai, L.Q. Huang, Y. Si, J.Y. Yu, B. Ding, Biomimetic and superelastic slica nanofibrous aerogels with rechargeable bactericidal function for antifouling water disinfection. ACS Nano 14(7), 8975$8984(2020)$

3. Z.C. Yin, Y.P. Pan, M.T. Bao, Y. Li, Superhydrophobic magnetic cotton fabricated under low carbonization temperature for effective oil/water separation. Sep. Purif. Technol. 266, 118535 (2021)

4. J.W. Xue, L. Zhu, X. Zhu, H. Li, C. Ma, S.F. Yu, D.F. Sun, F.J. Xia, Q.Z. Xue, Tetradecylamine-MXene functionalized melamine sponge for effective oil/water separation and selective oil adsorption. Sep. Purif. Technol. 259, 118106 (2021)

5. J.W. Lu, D.D. Xu, J.K. Wei, S. Yan, R. Xiao, Superoleophilic and flexible thermoplastic polymer nanofiber aerogels for removal of oils and organic solvents. ACS Appl. Mater. Interfaces 9(30) 25533-25541 (2017)

6. H.Z. Liu, B.Y. Geng, Y.F. Chen, H.Y. Wang, Review on the aerogel-type oil sorbents derived from nanocellulose. ACS Sustainable Chem. Eng. 5(1), 49-66 (2017)

7. B. Tansel, M. Lee, Removal of crude oil from highly contaminated natural surfaces with corexit dispersants. Journal of Environmental Management 247, 363-370 (2019)

8. S.S. Long, Y.C. Feng, Y.Z. Liu, L.L. Zheng, L.H. Gan, J. Liu, X.H. Zeng, M.N. Long, Renewable and robust biomass carbon aerogel derived from deep eutectic solvents modified cellulose nanofiber under a low carbonization temperature for oil-water separation. Sep. Purif. Technol. 254, 117577 (2021)

9. R.C. Prince, R.R. Lessard, J.R. Clark, Bioremediation of marine oil spills. Oil Gas Sci Technol 151(4), 495-512 (2004)

10. A. Jaggi, J.R. Radovic, L.R. Snowdon, S.R. Larter, T.B.P. Oldenburg, Composition of the dissolved organic matter produced during in situ burning of spilled oil. Org. Geochem. 138, 103926 (2019)

11. Y.S. Lee, Y.T. Lim, W.S. Choi, One-step synthesis of environmentally friendly superhydrophilic and superhydrophobic sponges for oil/water separation. Materials 12(7), 1182, (2019)

12. S.Y. Liu, J.T. Wang, Eco-friendly and facile fabrication of polyimide mesh with underwater superoleophobicity for oil/water separation via polydopamine/starch hybrid decoration. Sep. Purif. Technol. 250, 117228 (2020)

13. Z.X. Wang, M.C. Han, J. Zhang, F. He, S.Q. Peng, Y.X. Li, Investigating and significantly improving the stability of tannic acid (TA)-aminopropyltriethoxysilane (APTES) coating for enhanced oil-water separation. J. Membr. Sci. 593, 117383 (2020) 
14. H.C. Bi, X. Xie, K.B. Yin, Y.L. Zhou, S. Wan, L.B. He, F. Xu, F. Banhart, L.T. Sun, R.S. Ruoff, Spongy graphene as a highly efficient and recyclable sorbent for oils and organic solvents. Adv. Funct. Mater. 22, 4421-4425 (2012)

15. P.K. Huang, F. Wu, B. Shen, X.H. Ma, Y.Q. Zhao, M.H. Wu, J. Wang, Z.H. Liu, H.B. Luo, W.G. Zheng, Bioinspired lightweight polypropylene foams with tunable hierarchical tubular porous structure and its application for oil-water separation. Chem. Eng. J. 370, 1322-1330 (2019)

16. Y.Y. Tian, H.Z. Ma, Solvent-free green preparation of reusable EG-PVDF foam for efficient oil-water separation. Sep. Purif. Technol. 253, 117506 (2020)

17. Y.C. Yu, X.L. Shi, L. Liu, J.M. Yao, Highly compressible and durable superhydrophobic cellulose aerogels for oil/water emulsion separation with high flux. J. Mater. Sci. 56(3), 2763-2776 (2021)

18. C.X. Tang, P. Brodie, Y.Z. Li, N.J. Grishkewich, M. Brunsting, K.C. Tam, Shape recoverable and mechanically robust cellulose aerogel beads for efficient removal of copper ions, Chem. Eng. J. 392, 124821 (2020)

19. X.D. Cheng, S.Y. Zhu, Y.L. Pan, Y.R. Deng, L. Shi, L.L. Gong, Fire retardancy and thermal behaviors of Cellulose nanofiber/zinc borate aerogel. Cellulose 27, 7463-7474 (2020)

20. P.Y. Hu, J. Lyu, C. Fu, W.B. Gong, J.H. Liao, W.B. Lu, Y.P. Chen, X.T. Zhang, Multifunctional aramid nanofiber/carbon nanotube hybrid aerogel films. ACS Nano 14(1), 688-697 (2020)

21. J. Zhu, S.H. Lv, T.H. Yang, T. Huang, H. Yu, Q.H. Zhang, M.F. Zhu, Facile and green strategy for designing ultralight, flexible, and multifunctional PVA nanofiber-based aerogels. Adv Sustain Syst 4, $1900141(2020)$

22. Z.M. An, C.S. Ye, R.B. Zhang, P. Zhou, Flexible and recoverable SiC nanofiber aerogels for electromagnetic wave absorption. Ceram. Int. 45(17), 22793-22801 (2019)

23. Y. Si, J.Y. Yu, X.M. Tang, J.L. Ge, B. Ding, Ultralight nanofibre-assembled cellular aerogels with superelasticity and multifunctionality. Nat. Commun. 5, 5802 (2014)

24. Q.X. Fu, L.F. Liu, Y. Si, J.Y. Yu, B. Ding, Shapeable, underwater superelastic, and highly phosphorylated nanofibrous aerogels for large-capacity and high-throughput protein separation. ACS Appl. Mater. Interfaces 11(47), 44874-44885 (2019)

25. Z.C. Qian, Z. Wang, Y. Chen, S.R. Tong, M.F. Ge, N. Zhao, J. Xu, Superelastic and ultralight polyimide aerogels as thermal insulators and particulate air filters. J. Mater. Chem. A 6(3), 828-832 (2018)

26. J. Zhu, G. Sun, Facile fabrication of hydrophilic nanofibrous membranes with an immobilized metalchelate affinity complex for selective protein separation. ACS Appl. Mater. Interfaces 6(2) 925-932 (2014) 
27. M.F. Li, R. Xiao, G. Sun, Formation and morphology development of poly(butylene terephthalate) nanofibers from poly(butylene terephthalate)/cellulose acetate butyrate immiscible blends. Polym. Eng. Sci. 51(5), 835-842 (2011)

28. P.P. Zhang, D.D. Xu, R. Xiao, Morphology development and size control of PA6 nanofibers from PA6/CAB polymer blends. J. Appl. Polym. Sci. 132, 42184 (2015)

29. M.J. Zhu, G.B. Xu, M.F. Yu, Y.B. Liu, R. Xiao, Preparation, properties, and application of polypropylene micro/nanofiber membranes. Polym. Adv. Technol. 23(2), 247-254 (2012)

30. Q.Z. Liu, J.H. Chen, T. Mei, X.W. He, W.B. Zhong, K. Liu, W.W. Wang, Y.D. Wang, M.F. Li, D. Wang, A facile route to the production of polymeric nanofibrous aerogels for environmentally sustainable applications. J. Mater. Chem. A 6(8), 3692-3704 (2018)

31. Y.Z. Lin, L.B. Zhong, S. Dou, Z.D. Shao, Q. Liu, Y.M. Zheng, Facile synthesis of electrospun carbon nanofiber/graphene oxide composite aerogels for high efficiency oils absorption. Environ. Int. 128, 37-45 (2019)

32. Y.Q. Lu, Z.X. Niu, W.Z. Yuan, Multifunctional magnetic superhydrophobic carbonaceous aerogel with micro/nano-scale hierarchical structures for environmental remediation and energy storage. Appl. Surf. Sci. 480, 851-860 (2019)

33. H.Z. Sai, R. Fu, L. Xing, J.H. Xiang, Z.Y. Li, F. Li, T. Zhang, Surface modification of bacterial cellulose aerogels' web-like skeleton for oil/water separation. ACS Appl. Mater. Interfaces 7(13), 7373-7381 (2015)

34. K. Lee, J.S. Jur, D.H. Kim, G.N. Parsons, Mechanisms for hydrophilic/hydrophobic wetting transitions on cellulose cotton fibers coated using $\mathrm{Al}_{2} \mathrm{O}_{3}$ atomic layer deposition. J. Vac. Sci. Technol. A 30(1), 1-7 (2012)

35. J.T. Korhonen, M. Kettunen, R.H.A. Ras, O. Ikkala, Hydrophobic nanocellulose aerogels as floating, sustainable, reusable, and recyclable oil absorbents. ACS Appl. Mater. Interfaces 3(6) 1813-1816 (2011)

36. J.W. Lu, S. Yan, W. Song, K.I. Jacob, R. Xiao, Construction and characterization of versatile flexible composite nanofibrous aerogels based on thermoplastic polymeric nanofibers. J. Mater. Sci. 55(19), 8155-8169 (2020)

37. A.W. Chan, R.A. Whitney, R.J. Neufeld, Kinetic controlled synthesis of pH-responsive network alginate. Biomacromolecules 9(9), 2536-2545 (2008)

38. M.A. Shirgholami, M.S. Khalil-Abad, R. Khajavi, M.E. Yazdanshenas, Fabrication of superhydrophobic polymethylsilsesquioxane nanostructures on cotton textiles by a solution-immersion process. J. Colloid Interface Sci. 359(2), 530-535 (2011) 
39. Q.F. Zheng, Z.Y. Cai, S.Q. Gong, Green synthesis of polyvinyl alcohol (PVA)-cellulose nanofibril (CNF) hybrid aerogels and their use as superabsorbents. J. Mater. Chem. A 2(9), 3110-3118 (2014)

40. G.R.J. Artus, S. Jung, J. Zimmermann, H.P. Gautschi, K. Marquardt, S. Seeger, Silicone nanofilaments and their application as superhydrophobic coating. Adv. Mater. 18(20), 2758-2762 (2006)

41. Y.T. Wang, L.Y. Chen, H. Cheng, B.J. Wang, X.L. Feng, Z.P. Mao, X.F. Sui, Mechanically flexible, waterproof, breathable cellulose/polypyrrole/polyurethane composite aerogels as wearable heaters for personal thermal management. Chem. Eng. J. 402, 126222 (2020)

42. H. Cheng, Y.Z. Li, B.J. Wang, Z.P. Mao, H. Xu, L.P. Zhang, Y. Zhong, X.F. Sui, Chemical crosslinking reinforced flexible cellulose nanofiber-supported cryogel. Cellulose 25(1), 573-582 (2018)

43. Y. Si, X.Q. Wang, L.Y. Dou, J.Y. Yu, B. Ding, Ultralight and fire-resistant ceramic nanofibrous aerogels with temperature-invariant superelasticity. Science Advances 4, eaas8925 (2018)

44. H.W. Liang, Q.F. Guan, L.F. Chen, Z. Zhu, W.J. Zhang, S.H. Yu, Macroscopic-scale template synthesis of robust carbonaceous nanofiber hydrogels and aerogels and their applications. Angew. Chem. Int. Ed. 51(21), 5101-5105 (2012)

45. M. Xu, D.N. Futaba, T. Yamada, M. Yumura, K. Hata, Carbon nanotubes with temperature-invariant viscoelasticity from $-196^{\circ}$ to $1000^{\circ} \mathrm{C}$. Science $330(6009), 1364-1368(2010)$

46. Y. Liu, Y.P. Zhang, T.G. Liao, L. Gao, M. Wang, X. Xu, X.X. Yang, H. Liu, Boron nitride-nanosheet enhanced cellulose nanofiber aerogel with excellent thermal management properties. Carbohydr. Polym.

241,116425 (2020)

47. W.B. Zhang, Y.Z. Zhu, X. Liu, D. Wang, J.Y. Li, L. Jiang, J. Jin, Salt-induced fabrication of superhydrophilic and underwater superoleophobic PAA-g-PVDF membranes for effective separation of oilin-water emulsions. Angew. Chem. Int. Ed. 53(3), 856-860 (2014)

48. F. Jiang, Y.L. Hsieh, Amphiphilic superabsorbent cellulose nanofibril aerogels. J. Mater. Chem. A 2(18), 6337-6342 (2014)

49. Y.P. Su, Z.Y. Li, H.J. Zhou, S.H. Kang, Y.X. Zhang, C.Z. Yu, G.Z. Wang, Ni/carbon aerogels derived from water induced self-assembly of Ni-MOF for adsorption and catalytic conversion of oily wastewater. Chem. Eng. J. 402, 126205 (2020)

50. A. Mulyadi, Z. Zhang, Y.L. Deng, Fluorine-free oil absorbents made from cellulose nanofibril aerogels. ACS Appl. Mater. Interfaces 8(4), 2732-2740 (2016)

51. A. Li, H.X. Sun, D.Z. Tan, W.J. Fan, S.H. Wen, X.J. Qing, G.X. Li, S.Y. Li, W.Q. Deng, Superhydrophobic conjugated microporous polymers for separation and adsorption. Energ Environ Sci 4(6), 2062-2065 (2011) 
52. P. Song, J.W. Cui, J. Di, D.B. Liu, M.Z. Xu, B.J. Tang, Q.S. Zeng, J. Xiong, C.D. Wang, Q. He, L.X. Kang, J.D. Zhou, R.H. Duan, B.B. Chen, S.S. Guo, F.C. Liu, J. Shen, Z. Liu, Carbon microtube aerogel derived from kapok fiber: an efficient and recyclable sorbent for oils and organic solvents. ACS Nano 14(1), 595-60 (2020)

53. H.C. Bi, Z.Y. Yin, X.H. Cao, X. Xie, C.L. Tan, X. Huang, B. Chen, F.T. Chen, Q.L. Yang, X.Y. Bu, X.H. Lu, L.T. Sun, H. Zhang, Carbon fiber aerogel made from raw cotton: a novel, efficient and recyclable sorbent for oils and organic solvents. Adv. Mater. 25(41), 5916-5921 (2013)

\section{Figures}

(a)

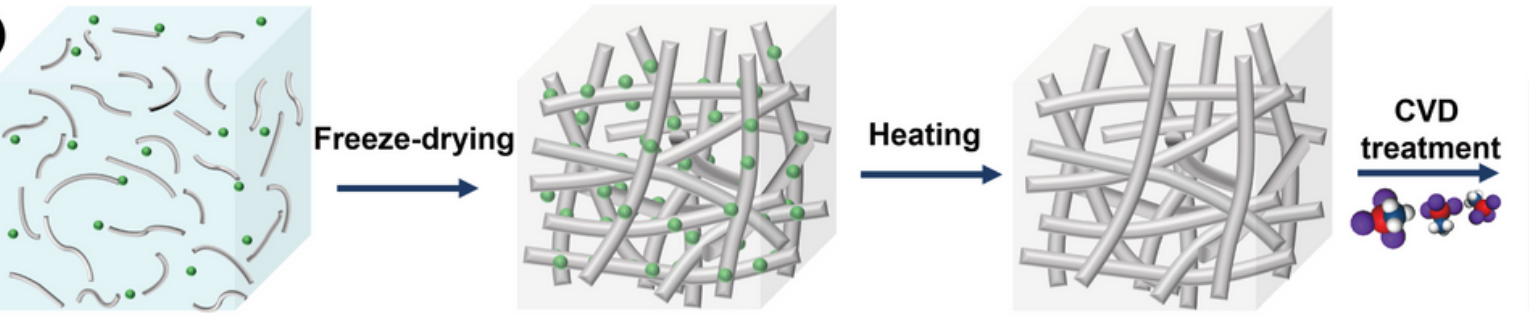

EVOH/GA nanofibrous suspension

Nanofibrous framework

EVOH NFAs

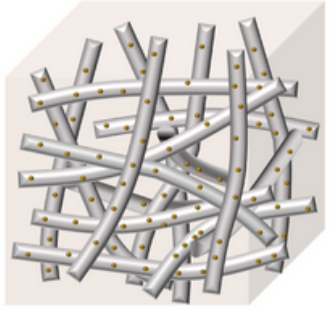

Silane-coated EVOH NFAs

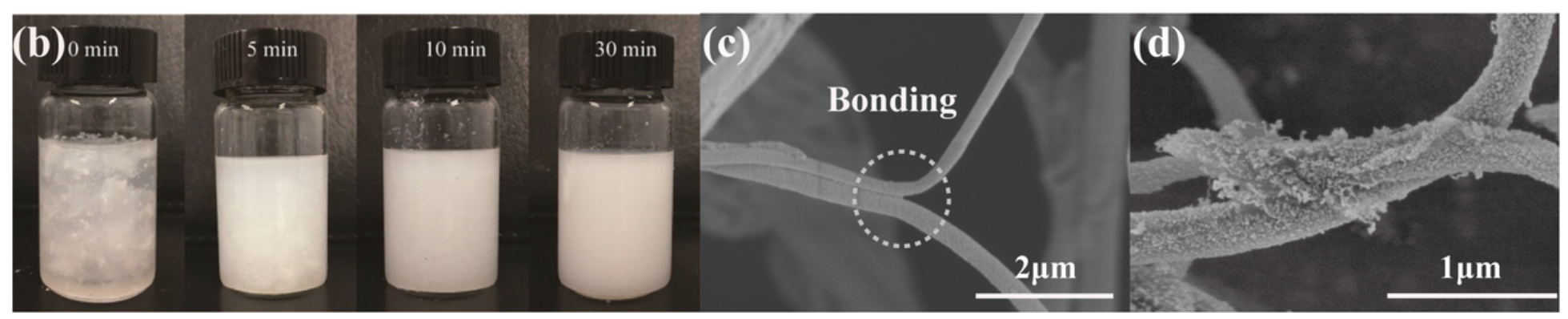

Figure 1

(a) Schematic illustration of the preparation pathway of silane-coated EVOH NFAs, (b) EVOH nanofibrous suspensions after various homogenization times, (c) the bonding among EVOH nanofibers, (d) morphology of siloxane coatings on the surface of EVOH nanofibers. 


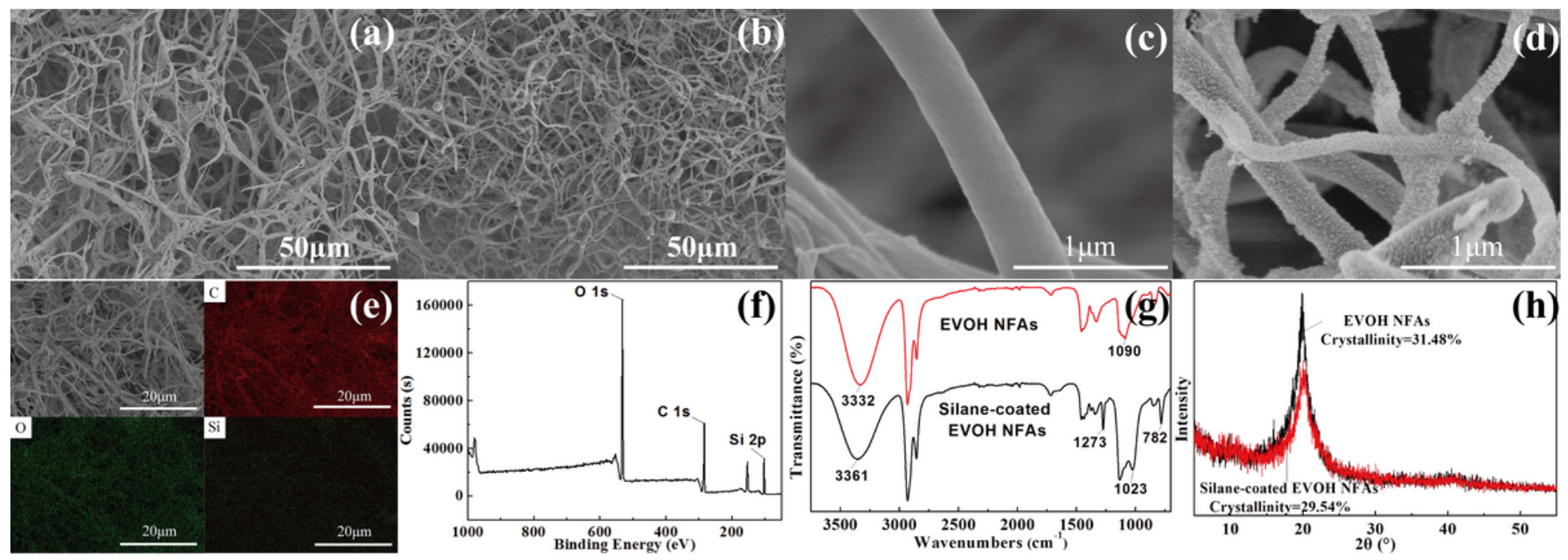

Figure 2

FE-SEM micrographs of $(a, c)$ EVOH NFAs and $(b, d)$ silane-coated EVOH NFAs, (e) the SEM-EDS elemental mapping images of silane-coated EVOH NFAs exhibiting the distribution of $\mathrm{C}, \mathrm{O}$ and $\mathrm{Si}$ in the same area, (f) XPS survey of silane-coated EVOH NFAs, (g) ATR-FTIR spectra and (h) XRD patterns of EVOH NFAs and silane-coated EVOH NFAs. 

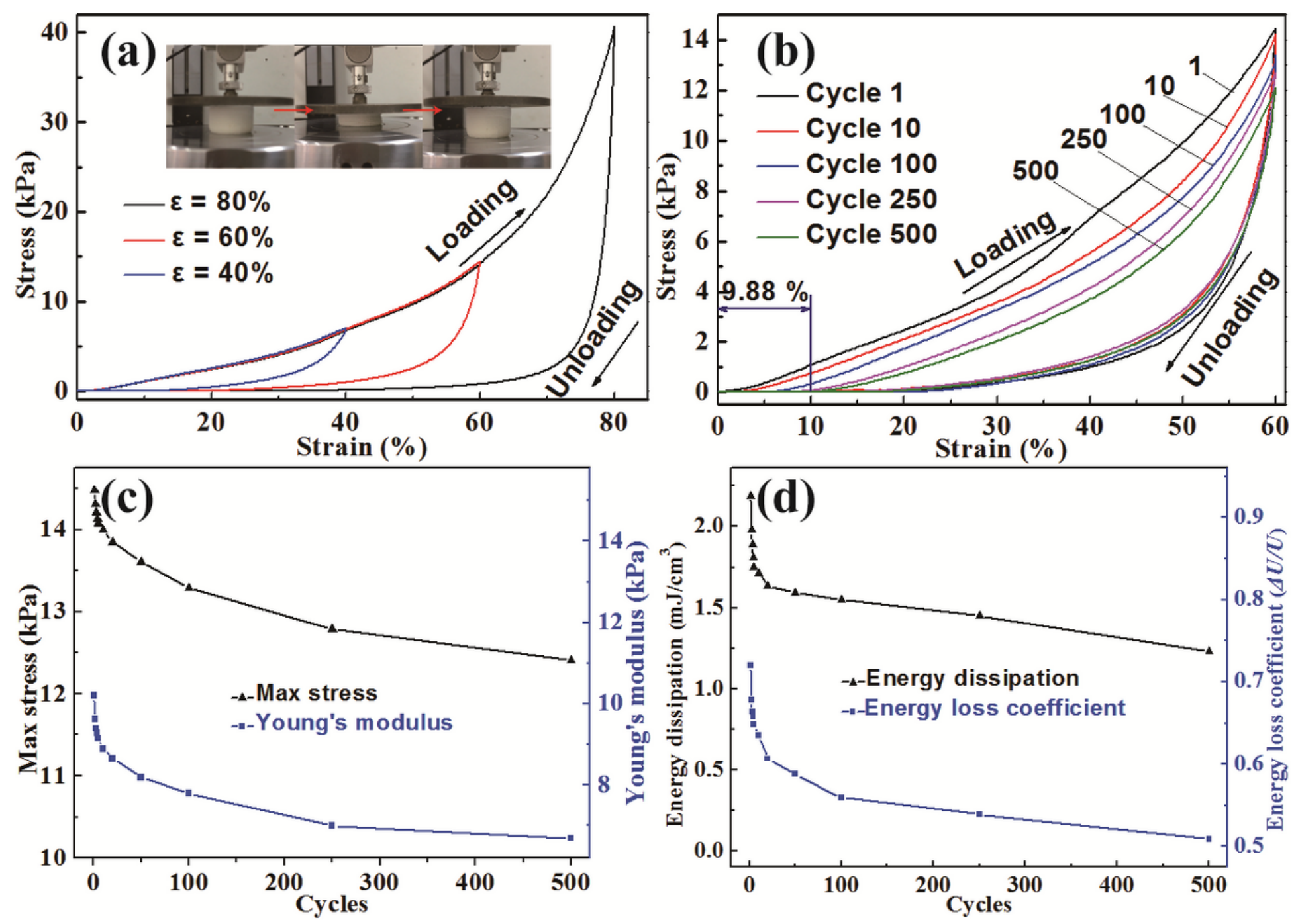

Figure 3

Mechanical properties of aerogels: (a) compressive $\sigma-\varepsilon$ curves of silane-coated EVOH NFAs at different strains $(\varepsilon=40,60,80 \%)$, (b) cyclic $\sigma-\varepsilon$ curves of silane-coated EVOH NFAs with $\varepsilon$ of $60 \%$, (c) the corresponding Young's modulus and maximum stress, (d) energy dissipation and energy loss coefficient as a function of compressive test cycles. 

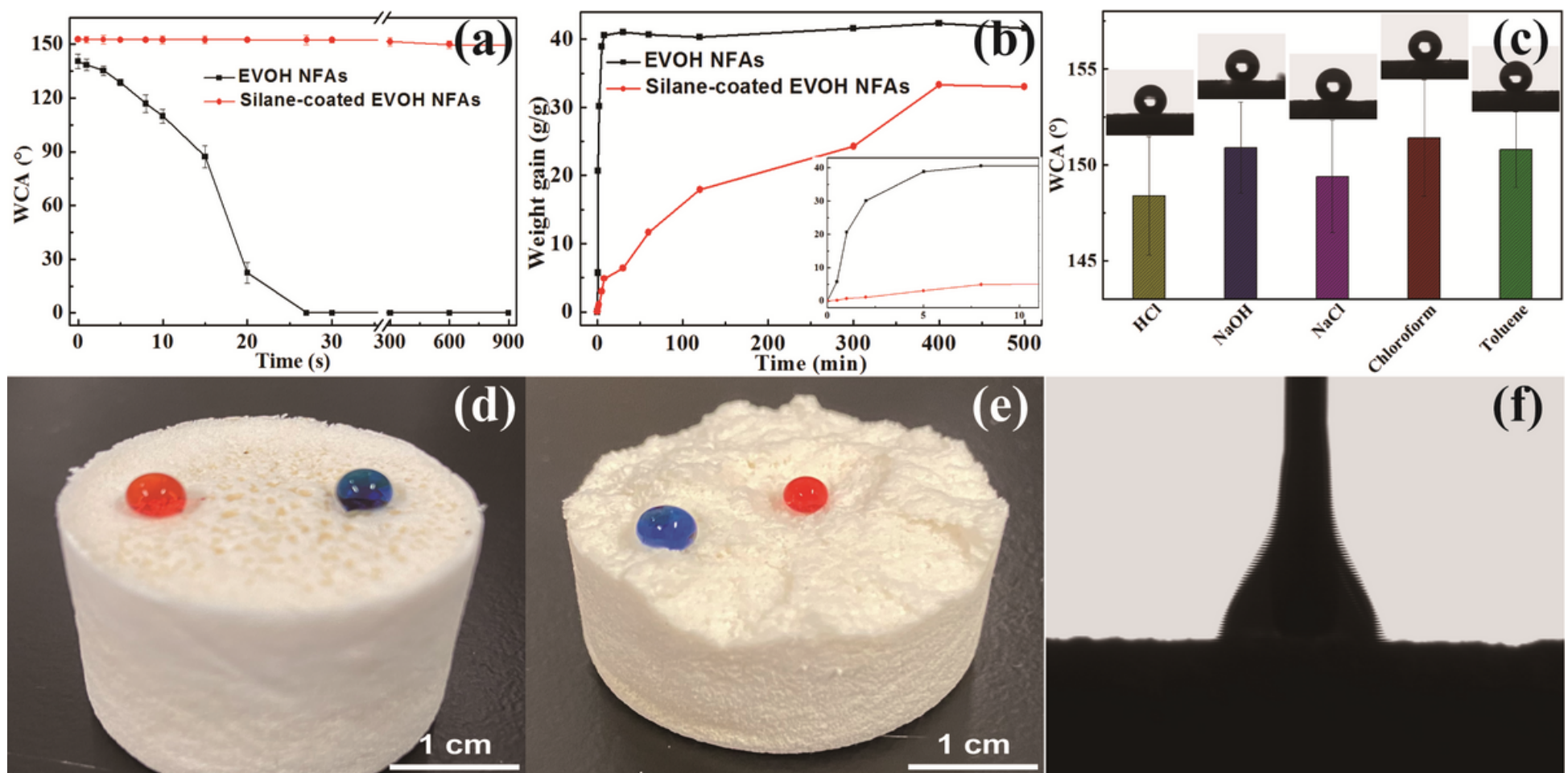

(f)

Figure 4

Surface wettability of NFAs: (a) the variation of WCA (b) water absorption of EVOH NFAs and silanecoated EVOH NFAs with increasing time, (c) WCA of silane-coated EVOH NFAs after immersing in corrosive medium liquids, salt solution and organic solvent, (d) stained water beading up (d) on the surface and (e) in the interior of silane-coated EVOH NFAs, (f) (c) OCA measurement of silane-coated EVOH NFAs. 


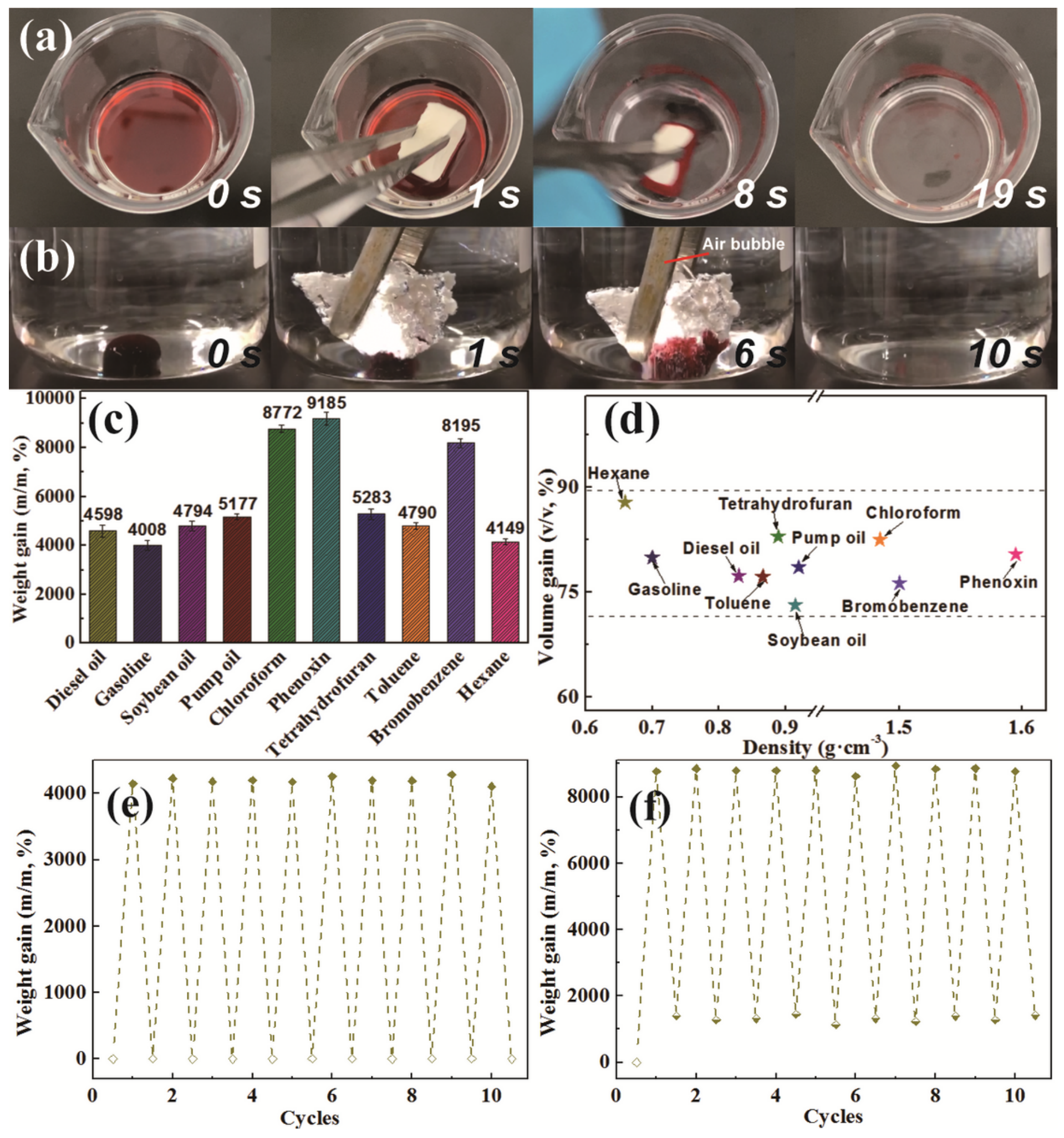

Figure 5

Surface wettability of NFAs: (a) the variation of WCA (b) water absorption of EVOH NFAs and silanecoated EVOH NFAs with increasing time, (c) WCA of silane-coated EVOH NFAs after immersing in corrosive medium liquids, salt solution and organic solvent, (d) stained water beading up (d) on the surface and (e) in the interior of silane-coated EVOH NFAs, (f) (c) OCA measurement of silane-coated EVOH NFAs. 


\section{Supplementary Files}

This is a list of supplementary files associated with this preprint. Click to download.

- SupplementaryMaterial.docx 